\title{
A scenario for leptogenesis at the TeV scale
}

\author{
Asmaa Abada*i \\ laboratoire de Physique theorique, \\ bât 210, Université Paris-Sud XI, \\ 91405 Orsay, France. \\ E-mail: abadaeth.u-psud.fr
}

\begin{abstract}
We propose a scenario of thermal leptogenesis at the $\mathrm{TeV}$ scale in the context of an extension of the Standard Model with 4 generations. We also add one right-handed Majorana neutrino for each generation to generate left handed neutrino masses via see-saw. The rôle of the fourth lepton doublet is not much different from that of a supersymmetric partner, so the leptogenesis consequences of a four generation scenario are indicators of a more generic picture. We obtain a value for the baryon asymmetry of the Universe in accordance with observations by solving the full set of coupled Boltzmann equations for this model.
\end{abstract}

International Europhysics Conference on High Energy Physics

July 21st - 27th 2005

Lisboa, Portugal

\footnotetext{
* Speaker.

$\dagger$ This talk is based on work done with M. Losada and H. Aissaou [1, 2]
} 
We have so far two experimental evidences to call for physics beyond the standard model (SM): the neutrino oscillations and the baryonic asymmetry of the universe (BAU). The SM with righthanded $(\mathrm{RH})$ neutrinos $(v)$ provides an elegant mechanism for thermal leptogenesis. There are two intertwined requirements: reproduce the spectrum for light $v$ in the observed range and generate enough CP asymmetry through the out-of-equilibrium decay of heavy $\mathrm{RH} v$ ([3]). This asymmetry can be transmitted to the baryonic sector through sphaleron induced processes to explain the BAU. To achieve these two requirements, the RH $v$ should have Majorana masses and they should couple to the left-handed (LH) lepton doublets and the SM Higgs via complex Yukawa. The scenario of leptogenesis occurring at the $\mathrm{TeV}$ scale has been investigated for example in references ([1,2],[4][6]). This scale is accessible in ongoing and near future colliders. Moreover, interesting new physics (like supersymmetry, extra dimensions, etc) could be revealed around that scale. It is now known that with $3 \mathrm{RH}$ neutrinos it is difficult to achieve $\mathrm{TeV}$ scale leptogenesis and reproduce at the same time the small LH $v$ masses [1], unless one considers quasi-degenerate Majorana $v$ [4] or small fine-tuning [5]. There are two approaches in the literature to realise $\mathrm{TeV}$ scale leptogenesis: (a) consider a quasi-degenerate spectrum of heavy RH $v$ and enhance $\mathrm{CP}$ asymmetry through resonant effects [7]; (b) extend the phase space parameters, either (i) by admiting, for example, extra couplings that allow three body decays of the RH $v$ leading to an enhancement of $\mathrm{CP}$ asymmetry [8], or, (ii) by extending the particle content. As regards the latter possibility, one may adopt among others either of two approaches: (1) consider a supersymmetric framework [9], (2) minimally extend the SM by having a fourth chiral generation and add a heavy RH $v$ for each of the four generations, assuming that the lepton asymmetry is due to the decay of the lightest $\mathrm{RH}$ $v\left(N_{1}\right)$ in the TeV scale $[1,2]$. The scenario we present here is precisely this last approach. It is a simple extension of the Fukugita-Yanagida model [3] including one more generation to the SM. We will show how this simple model which can explain the BAU and provide appropriate values for $v$ masses and mixing angles. It is based on the out-of-equilibrium decay of a $\mathrm{RH} v$, in a $\mathrm{C}$ and $\mathrm{CP}$ violating way, such that a leptonic asymmetry is produced. The latter is converted into a BAU due to $(B+L)$-violating sphaleron interactions which are in equilibrium above the electroweak breaking scale.

\section{The scenario and its constraints}

The relevant part of the Lagrangian of the model we consider is given by,

$$
L=L_{S M}+\bar{\psi}_{R_{i}} i \partial / \psi_{R_{i}}-\frac{M_{N_{i}}}{2}\left(\bar{\psi}_{R_{i}}^{c} \psi_{R_{i}}+\text { h.c. }\right)-\left(\lambda_{i \alpha}^{v} \bar{L}_{\alpha} \psi_{R_{i}} \phi+\text { h.c. }\right),
$$

where $\psi_{R_{i}}$ are 2-component spinors describing the RH neutrinos and we define a Majorana 4component spinor, $N_{i}=\psi_{R_{i}}+\psi_{R_{i}}^{c}$. Our index i runs from 1 to 4 , and $\alpha=e, \mu, \tau, \sigma$. The $\sigma$ component of $L_{\alpha}$ corresponds to a LH lepton doublet which must satisfy the LEP constraints from the Z- width on a fourth LH $v$ [10]. The $\lambda_{i \alpha}^{v}$ are Yukawa couplings and the field $\phi$ is the SM Higgs boson doublet with vev $v$. We work in the basis in which the mass matrix for the RH $v M$ is diagonal and real, $M=\operatorname{diag}\left(M_{1}, M_{2}, M_{3}, M_{4}\right)$ and define $m_{D}=\lambda^{v} v$. To leading order the induced see-saw $v$ mass matrix for the LH $v$ is given by, $m_{v}=\lambda^{v^{\dagger}} M^{-1} \lambda^{v} v^{2}$ which is diagonalized by the well known PMNS matrix.

We consider the out-of-equilibrium decay of the lightest of the gauge singlets $N_{1}$. There are two key points: (a) the CP asymmetry 


$$
\varepsilon_{1}=\frac{1}{8 \pi\left[\lambda \lambda^{\dagger}\right]_{11}} \sum_{j \neq 1} \operatorname{I} m\left[\lambda \lambda^{\dagger}\right]_{1 j}^{2} f\left(M_{N_{j}}^{2} / M_{N_{1}}^{2}\right)
$$

where $f$ is a loop factor, has to be magnified by the presence of a large Yukawa coupling, and (b) the condition of out-of-equilibrium decay of $N_{1}$ has to be ensured, i.e., $\Gamma_{N_{1}}=\left(\lambda^{\dagger} \lambda\right)_{11} M_{1} / 8 \pi=$ $\sum_{\alpha=1}^{4} \frac{\left(\lambda_{\alpha 1}^{*} \lambda_{\alpha 1}\right)}{M_{1}} / 8 \pi \equiv \tilde{m}_{1}^{(4 G)} M_{1}^{2} /\left(8 \pi v^{2}\right)<H\left(T=M_{N_{1}}\right)$, where $H$ is the Hubble expansion rate. These conditions restrict the size of the Yukawa couplings.

Another feature of our scenario is that the Yukawa coupling of the heavy LH neutrino is unsupressed (order 1) to ensure that the masses of the fourth generation leptons are heavy enough, and also to give an enhancement to the value of the CP-asymmetry (1.2). However, we note that the $\Delta L=2$ processes involving the fourth family of leptons in external legs are rapid and hence in thermal equilibrium. Consequently, the lepton asymmetry in the fourth leptonic direction is washed out. This changes the chemical equilibrium equations such that the relationship between $B$ and $L$ is modified to be: $Y_{B}=-(8 N+4 /(14 N+25)) \sum_{\alpha=e, \mu, \tau} Y_{L_{\alpha}}$, where $Y_{L}$ is the produced leptonic asymmetry only for the light active flavours.

\section{Boltzmann Equations and Results}

The evolution of the abundance of the $N_{1}, Y_{N_{1}}$, and the lepton asymmetry $Y_{L}$ are given by

$$
\begin{aligned}
\frac{d Y_{N_{1}}}{d z} & =-\frac{z}{s H\left(M_{1}\right)}\left(\frac{Y_{N_{1}}}{Y_{N}^{e q}}-1\right)\left(\gamma_{D_{1}}+\gamma_{S_{1}}\right), \\
\frac{d Y_{L}}{d z} & =-\frac{z}{s H\left(M_{1}\right)}\left[\varepsilon_{1} \gamma_{D_{1}}\left(\frac{Y_{N_{1}}}{Y_{N}^{e q}}-1\right)+\gamma_{W} \frac{Y_{L}}{Y_{L}^{e q}}\right],
\end{aligned}
$$

where $z=\frac{M_{1}}{T}, s$ is the entropy density and $\gamma_{D_{j}}, \gamma_{S_{j}}$ are the interaction rates for the decay and $\Delta L=$ 1 scattering contributions, respectively. $\gamma_{W}$ is a function of $\gamma_{D_{j}}$ and $\gamma_{S_{j}}$ and $\Delta L=2$ interaction rate processes, called the washout factor which is responsible for the damping of the produced asymmetry. In eqs. (2.1) and (2.2), $Y_{i}^{e q}$ is the equilibrium number density of a particle $i$. Explicit expressions of the interaction rates $\gamma_{D_{j}}, \gamma_{S_{j}}$ and $\gamma_{W}$ are given in details in [2]. An important comment is in order. The net leptonic asymmetry is produced only in the first 3 flavours as the strong $\Delta L=2$ process involving the fourth generation washes out the asymmetry in this direction.

In figure 1, we illustrate for a given set of input parameters the different thermally averaged reaction rates contributing to $\mathrm{BE}$ as a function of $z=\frac{M_{1}}{T}: \Gamma_{X}=\frac{\gamma_{X}}{n_{N_{1}}^{e q}}, X=D, S, \Delta L=2$. It is clear from this plot that for this set of parameters, and this is true for a wide range of parameter space, all rates at $z=1$ fulfill the out-of-equilibrium condition (i.e. $\Gamma_{X}<H(z=1)$ ), and so the expected washout effect due to the $\Delta L=2$ processes will be small. The generated value of the BAU is $\eta_{B} \simeq 6 \times 10^{-10}$. Applying the see-saw mechanism to our model for the chosen values of the parameters, we obtain $m_{v_{4}}>48 \mathrm{GeV}$, and the three light $v$ masses are of the order of $10^{-1} \mathrm{eV}$ to a few $10^{-7} \mathrm{eV}$. 

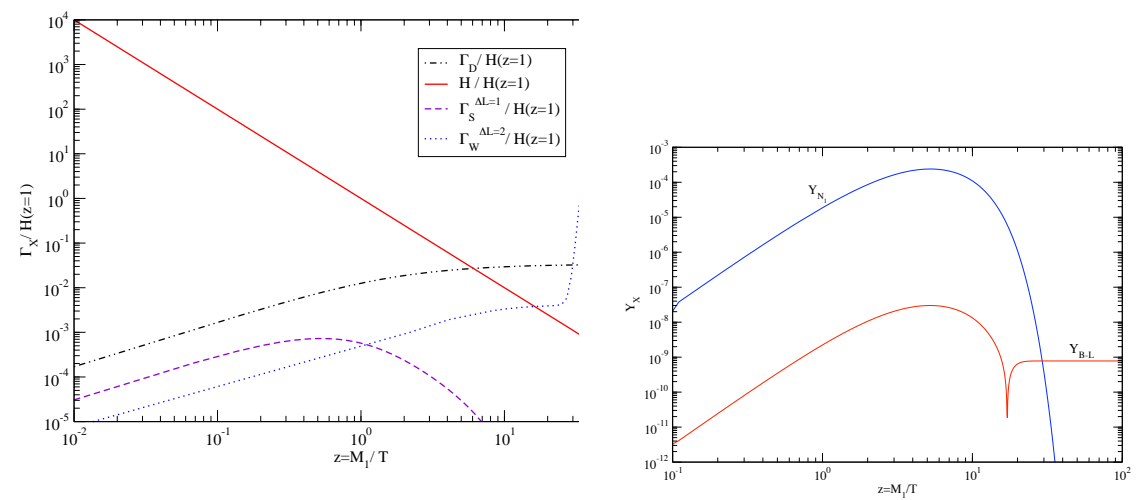

Figure 1: Thermally averaged decay rates normalized to the expansion rate of the Universe $H(z=1)($ left). Abundance $Y_{N_{1}}$ and the baryon asymmetry $Y_{B-L}$ (right).

\section{Conclusions}

This scenario is an extension of the SM by invoking a fourth chiral family, the leptogenesis effect of which is a generic indicator of many a physics beyond the SM, e.g., supersymmetry. We have presented the solutions to the coupled system of BE for our TeV scale model of thermal leptogenesis. We have carefully considered the effect of the interactions involving the heavy fourth generation leptonic fields and consistently written the BE which contribute to the final BAU together with the appropriate conversion factor from the lepton asymmetry to the baryonic one. Our results show that in this simple extension of the SM it is possible to produce the right amount of the BAU in a generic way.

\section{References}

[1] A. Abada and M. Losada, Nucl. Phys. B 673 (2003) 319 [arXiv:hep-ph/0306180];

[2] A. Abada, H. Aissaoui and M. Losada, Nucl. Phys. B 728 (2005) 55; arXiv:hep-ph/0406304.

[3] M. Fukugita and T. Yanagida, Phys. Lett. B 174 (1986) 45.

[4] A. Pilaftsis, Phys. Rev. D 56 (1997) 5431; G. C. Branco et al., , arXiv:hep-ph/0507092; R. Gonzalez Felipe et al., Phys. Rev. D 70 (2004) 085009; A. Pilaftsis and T. E. J. Underwood, arXiv:hep-ph/0506107.

[5] L. Boubekeur, T. Hambye and G. Senjanovic, Phys. Rev. Lett. 93 (2004) 111601.

[6] M. Senami and K. Yamamoto, Phys. Rev. D 69 (2004) 035004;T. Hambye et.al, JHEP 0407 (2004) 070 .

[7] A. Pilaftsis and T. E. J. Underwood, Nucl. Phys. B 692 (2004) 303 [arXiv:hep-ph/0309342].

[8] T. Hambye, Nucl. Phys. B 633 (2002) 171 [arXiv:hep-ph/0111089].

[9] G. F. Giudice et al., Nucl. Phys. B 685 (2004) 89, and references therein; J. Ellis and S. K. Kang, arXiv:hep-ph/0505162; J. R. Ellis, M. Raidal and T. Yanagida, Phys. Lett. B 581 (2004) .

[10] See the LEP Electroweak Working Group webpage:http://lepewwg.web.cern.ch/LEPEWWG/. 